\title{
Regulation of catecholamine synthesizing enzyme gene expression in human pheochromocytoma
}

\author{
H Lehnert \\ Klinik für Endokrinologie und Stoffwechselkrankheiten, Zentrum für Innere Medizin, Otto-von-Guericke Universität, Leipziger Strasse 44, \\ 39120 Magdeburg, Germany
}

This issue of the European Journal of Endocrinology contains an important report investigating the expression of the mRNAs coding for catecholamine synthesizing enzymes in human pheochromocytomas (1). Despite all efforts to characterize these tumors by molecular biological techniques and to study the expression of various growth factors and their receptors, investigations into the genuine enzymes have so far been surprisingly scarce. This and other studies clearly suggest that this methodological approach cannot only be used to further characterize the genomic properties with regard to the expression of the various enzymes in these tumors, but also to yield prognostic criteria in terms of benign versus malignant pheochromocytoma. In order to fully appreciate the pathophysiological relevance and implications of this approach, it is mandatory to characterize the nature of this tumor and basic mechanisms of catecholamine biosynthesis. I shall thus use this editorial to briefly review the clinical genetics and aspects of pheochromocytoma, followed by novel general aspects of catecholamine enzyme regulation, as well as synthesis, storage and secretion of these hormones, and finally discuss some of the data available on catecholamine synthesizing enzyme mRNA expression in human pheochromocytomas and their possible clinical relevance.

The pheochromocytoma represents a catecholamine secreting tumor that can arise both from intra- and extraadrenal chromaffin cells. The term paraganglioma describes a subgroup of those tumors which originate in extraadrenal chromaffin cells. These cells are of neuroectodermal origin and thus part of the so-called diffuse neuroendocrine system. This embryogenetic basis also explains the association of a pheochromocytoma with other disorders sharing this origin. There are few data on the incidence and prevalence of this tumor; a prevalence between 0.2 and $0.4 \%$ in patients with diastolic hypertension is suggested.

A pheochromocytoma can be classified according to various categories. A major criterion is the sporadic versus familial appearance; another is represented by localization (intra- versus extraadrenal). Finally, benign and malignant pheochromocytomas have to be differentiated. Approximately $10 \%$ of all pheochromocytomas of the adult reveal an extraadrenal localization, while this number appears to be higher in children, with approximately 35\%. The majority of pheochromocytomas (approximately 90\%) are of sporadic nature; familiarity has in particular been observed when the following diseases are either underlying or coexisting: multiple endocrine neoplasia (MEN) type $2 \mathrm{a} / \mathrm{b}$, von Hippel-Lindau syndrome (vHLS) and neurofibromatosis type I (NFI). In addition to NFI, tuberosis sclerosis and Sturge-Weber disease are of neuroectodermal origin and associated with pheochromocytoma. As mentioned, the potential malignancy of these tumors represents a major clinical problem. Approximately $20 \%$ of all pheochromocytomas will eventually be malignant. The most strict criterion for malignancy is the appearance of metastases in organs that otherwise do not contain chromaffin cells (2).

The pathogenesis of pheochromocytoma is still unclear. It is only the pheochromocytoma within the context of MEN, vHLS or NF that possesses a characterized molecular genetic background. In MEN 2a/b, mutations of the RET proto-oncogene and chromosome 10 have been very well described. This oncogene belongs to the group of tyrosine kinase receptor coding oncogenes, which can be activated by DNA rearrangement (3). The tyrosine kinase then functions as a receptor for the glial cell-derived neurotrophic factor (GDNF) and GDNF-receptor complex. The vHLS also represents a hereditary disease, which is characterized by the appearance of a number of benign and malignant tumors. The leading clinical characteristics are angioblastomas, retinal angiomas, renal cell tumors and pheochromocytomas, the last of these occurring with a frequency of 15 to $25 \%$. The vHLS is further differentiated into type 1 , type $2 \mathrm{a}$ and type $2 \mathrm{~b}$, where pheochromocytoma is associated with types $2 \mathrm{a}$ and $\mathrm{b}$. The vHLS suppressor gene is located on chromosome 3p 25-26. In NFI, a pheochromocytoma is observed in approximately $5 \%$ of cases; vice versa in 1 to $2 \%$ of all cases diagnosis of a pheochromocytoma will eventually also lead to the diagnosis of NFI. The NFI gene codes for the protein neurofibromin, which stimulates the GTPase activity of $\mathrm{p} 21$ ras in vitro. It is thus conceivable that signal transduction will be induced via this protein. The NFI gene also most likely represents a tumor suppressor gene with the mutated gene located on chromosome 17. 
In sporadic, non-familial pheochromocytoma the pathogenesis is - as mentioned above - as yet unknown. Pheochromocytomas contain receptors for numerous growth factors, such as insulin-like growth factor-I (IGF-I) and IGF-II. Moreover, high affinity somatostatin receptors are expressed, typically type II and IV. The somatostatin receptor-positive tumors exhibit loss of heterozygozity on chromosome 1p. Similarly, on chromosomes 3p, 17p and 23q further allele losses have been described. It is conceivable that these mutations affect tumor suppressor genes and thus play a role in tumor development and manifestation.

As indicated above, I will briefly review novel aspects of catecholamine biosynthesis that are relevant to the understanding of these processes in pheochromocytoma tumor tissue. Catecholamines are consecutively synthesized by a series of enzymes, which are localized in both the cytoplasm and vesicles of neuronal and chromaffin cells. Dependent on the presence of either dopamine-betahydroxylase $(\mathrm{DBH})$ or phenylethanolamine-N-methyltransferase (PNMT) the synthesis will lead to either noradrenaline or adrenaline (4). The precursor of catecholamine synthesis is the large neutral amino acid L-tyrosine, which is either taken in with food or is a product of the hepatic conversion of phenylalanine. L-Tyrosine is hydroxylated to 3,4dehydroxyphenylalanine (L-DOPA) by the rate-limiting enzyme tyrosine hydroxylase (TH). This enzyme possesses a $K_{\mathrm{m}}$ of $2 \times 10^{-5} \mathrm{~mol} / \mathrm{l}$ in the adrenal medulla and $0.4 \times 10^{-5} \mathrm{~mol} / \mathrm{l}$ in the central nervous system (CNS). It is the product of a single, multiple-exonic gene. While in most species the translation products from a single mRNA species combine to form a holoenzyme, in humans, alternative splicing of human TH RNA results in three additional RNA forms. The hydroxylation of the phenol ring using molecular oxygen, $\mathrm{Fe}^{2}$ and reduced pteridine (tetrahydrobiopterin, TH4) as cofactors is the rate-limiting step in catecholamine biosynthesis. TH4 serves as donor for hydrogen atoms, maintaining $\mathrm{TH}$ in a reduced active state. Catechols inhibit $\mathrm{TH}$ by oxidation of $\mathrm{TH} 4$ to pteridine and thus block the donation of hydrogen and subsequently the activation of TH. Moreover, catechols act as competitive inhibitors of $\mathrm{TH} 4$ at the catalytic binding site of TH. Under circumstances of enhanced neuronal activity TH may be activated by phosphorylation of multiple serine sites on the enzyme through different protein kinases $(5,6)$. Prolonged stimulation leads to a slow and long-lasting induction of the $\mathrm{TH}$ enzyme protein subsequent to activation of $\mathrm{TH}$ gene transcription and induction of TH mRNA. Depending on the localization of phosphorylation, either $V_{\max }$ or the affinity of $\mathrm{TH}$ for the cofactor are increased. Negative feed-back, leading to TH dephosphorylation, is mediated via alpha 2 adrenergic or $\mathrm{D}_{2}$ dopaminergic receptors, which may activate cAMP or $\mathrm{Ca}^{2} /$ calmodulin-dependent protein phosphatases. Numerous other neurotransmitters, including acetylcholine, which are released from preganglionic neurons and interact with receptors on the postganglionic neurons of sympathetic ganglia or the chromaffin cells play an important role in regulating TH activity (7).

The next step in catecholamine synthesis is represented by the conversion of L-DOPA to dopamine, catabolized by the cytosolic aromatic L-amino acid decarboxylase (AAD, DOPA-decarboxylase) with a $K_{\mathrm{m}}$ of $4 \times 10^{-4} \mathrm{~mol} / \mathrm{l}$. Pyridoxal phosphate (vitamin B6) is used as a cofactor. This is the final step of catecholamine synthesis in dopaminergic neurons. The enzyme is expressed both in monoamine producing neuronal cells as well as in kidney, liver and thymic cells. The rat $\mathrm{AAD}$ is encoded by a single gene in both these neuronal and non-neuronal tissues.

In noradrenergic and adrenergic neurons, as well as in chromaffin cells of the adrenal medulla, dopamine is catalyzed to noradrenaline via DBH. In addition to dopamine, this $\mathrm{Cu}^{2}$-containing enzyme also betahydroxylases several other phenylethylamines. DBH is a mixed-function oxidase, which utilizes molecular oxygen and ascorbate as hydrogen donors.

Within the chromaffin cells of the adrenal medulla and, additionally, in some CNS adrenergic neurons the enzyme PNMT catalyzes the N-methylation of noradrenaline to adrenaline. This enzyme is localized in the cytosol and requires S-adenosylmethionine as a methyl donor. This enzyme is not substrate specific and catalyzes the methylation of several phenylethanolamine derivatives. Most important, also for the understanding of key data of studies on catecholamine synthesizing enzymes in various pheochromocytomas, is the induction of PNMT activity through glucocorticoids (8) (Fig. 1).



Figure 1 Pathway of catecholamine biosynthesis including cofactors (for abbreviations see text). 
To briefly summarize mechanisms of storage and secretion: the uptake of catecholamines into the granules of chromaffin cells is mediated by vesicular monoamine transporters. The energy required results from a hydrogen ion gradient which is maintained through an $\mathrm{H}^{+}$-ATPase. The granules of the adrenal medullary cells contain catecholamines and ATP in a $4: 1$ ratio; this ratio is $6-8: 1$ in the vesicles of noradrenergic neurons. It is assumed that the anionic phosphate groups of ATP interact in a reciprocal fashion with catecholamines, which under physiological conditions behave as cations; the result is an enhanced binding of catecholamines in the intracellular vesicles. At least partially, catecholamines are complex bound with chromogranin A. This will eventually avoid high osmotic pressure within the granules. The physiological relevance of this compartmentalization is the safe storage of catecholamines until secretion is mediated by a physiological stimulus, and thus protection against metabolic enzymes.

Secretion of catecholamines from the chromaffin cells of the adrenal medulla is mainly mediated by acetylcholine, which through nicotinergic receptors at the chromaffin cell membrane leads to a change in the membrane permeability for calcium and other ions (7). Supposedly, the calcium influx represents the relevant stimulus for adrenaline release. In addition, adrenaline secretion may also be stimulated through histamine receptors, $\mathrm{K}^{+}$ions, bradykinin and other neuromodulators via enhanced calcium influx.

While the physiological secretion of catecholamines is controlled and regulated in a very tight manner, pheochromocytomas very often secrete their hormone end products, i.e. adrenaline and noradrenaline, in an unregulated and sometimes bizarre fashion. Thus, a substantial secretion of dopamine is not readily detected and there is a great variation in both the immunohistochemical detection of TH, PNMT and, subsequently, the relative ratio of noradrenaline to adrenaline. The tissue content of catecholamines in general appears to be very high. Numerous studies have demonstrated that the rate-limiting enzyme TH is very active in pheochromocytomas, as shown in enzyme kinetic studies both in pheochromocytomas and in PC1 2 cultures, a pheochromocytoma cell line derived from rats. Moreover, cell studies suggest that the increase in the biosynthesis of catecholamines may follow an overexpression of the TH gene. Unfortunately, the experiments performed to study the activity and expression of catecholamine synthesizing enzymes in pheochromocytomas have in general been scarce. Until recently, most reports focused on the immunohistochemical localization of these enzymes in tumor tissue. Thus, in one study it was shown that the relevant enzymes TH, AAD, DBH and PNMT were readily detected in functioning pheochromocytoma (9). On the other hand, 'non-functioning' pheochromocytomas had very few cells immunoreactive to TH. These findings were obtained regardless of whether a so-called functioning tumor was found at an adrenal or extraadrenal site. The study now appearing in this issue (1) went on to further characterize the mechanisms controlling catecholamine synthesis in pheochromocytomas by measuring the mRNA levels of the relevant enzymes described above. These authors were able to demonstrate that the enzyme mRNA levels were much higher in the pheochromocytoma tumor tissue than in the normal adrenal medullas. There was a strong positive correlation between the TH mRNA expression and the catecholamine content, with the mean content of catecholamines being no different in pheochromocytomas and normal adrenals. This clearly, and very rightly so, points to the regulation of catecholamine production at the level of gene expression. Also, since several other studies reported a higher activity of the TH enzyme, it clearly appears that TH mRNA expression also controls TH activity. This mechanism may thus be more relevant than the ones operating in normal tissue, where the phosphorylation of the enzyme protein is regulated by end product feed-back control. I will turn to these mechanisms, which are highly relevant for the understanding of enzyme activity and control of catecholamine synthesis, later in this review. While TH mRNA expression is higher in the pheochromocytoma tissue, the levels of PNMT mRNA have been found to be lower in the normal adrenals. This difference in enzyme mRNA expression has been found to be responsible for the difference in catecholamine content between tumor and normal adrenal tissue. Thus, tumor tissue low in PNMT mRNA expression will predominantly show a higher noradrenaline than adrenaline content and, subsequently, a higher noradrenaline secretion in the affected patient.

In a similar study, although based on a lower number of tumors, the mRNA of these enzymes was studied in six sporadic pheochromocytomas, two pheochromocytomas in vHLS, one in MEN $2 \mathrm{~b}$ and in one malignant extraadrenal tumor (10). The data obtained in this study in general resemble the ones by Isobe et al. (1). Thus, there was a very high correlation between TH mRNA and total catecholamine content. Moreover, the TH mRNA correlated well with the TH activity measured by a coupled decarboxylase technique. The results differed in one respect, namely that the catecholamine contents measured in the normal tissue were found to be clearly lower than in the tumor tissue. Further studies are certainly necessary to resolve this discrepancy. Very interestingly though, the TH mRNA levels were very different according to tumor type and underlying disease. Thus, the single MEN $2 b$ pheochromocytoma study had the highest TH mRNA levels, while these were very low in the tumors associated with vHLS. Also, the malignant extraadrenal pheochromocytoma had extremely low levels of TH mRNA, which is in general compatible with a dedifferentiation of tumor tissue. When a ratio of $\mathrm{TH}$ activity to catecholamine 
content was calculated, these were found to be lower in the pheochromocytoma as opposed to the normal adrenal tissue. This clearly suggests that in tumor tissue small increases in TH activity result in a much larger increase in catecholamine levels. TH activity was found to correspond with TH mRNA. In summary, the data from this report also support the notion that catecholamine content (and secretion under clinical conditions) is primarily controlled at the level of gene expression.

Accordingly, the mRNA levels of AAD and DBH were also found to be higher in the pheochromocytoma tissue. Since the tumor tissue analyzed in this study originated from presumably benign pheochromocytomas, results might have been very different in malignant tumors, where loss of DBH activity has been reported. Similarly, in another study (9) cells immunoreactive for $\mathrm{DBH}$ were much smaller in number in so-called non-functioning pheochromocytoma. Nonfunctioning and thus non-secreting tumor cells are more often found in malignant species. As mentioned briefly above, PNMT induction is a result of a physiological glucocorticoid environment. In the study by Isobe et al. (1) the PNMT mRNA levels were found to be higher in normal adrenals, corresponding with a preferential formation of noradrenaline versus adrenaline. In another study, PNMT was detected by immunohistochemical means in adrenal and extraadrenal pheochromocytoma (11) and in the so-called mixed type pheochromocytomas (9). This possible discrepancy also deserves further elucidation, but it may be speculated that the clearly decreased exposure to circulating glucocorticoids may not only be responsible for an altered enzyme activity, but also for a lowered gene expression of this enzyme. Most interestingly though, the synopsis of data from these studies suggests that differences on both the genomic and immunohistochemical level in terms of enzyme expression clearly exist and may account not only for the functioning versus non-functioning but also for the benign versus malignant character of this tumor. In this context, further studies evaluating the distribution of enzyme immunoreactivity across the tumor tissue may be important. Moreover, the determination of the enzyme isoforms also appears to be of particular relevance. Thus it has been shown that due to alternative splicing of $\mathrm{TH}$ mRNA four different forms of TH mRNA exist (12). The relative abundance of these isoforms has been shown to be similar in different tumor cell lines, but the immunocytochemical analysis demonstrated that immunoreactivity was unequally distributed among these cells. There was a direct relationship between the percentage of the cells positively stained for $\mathrm{TH}$ immunoreactivity and overall TH levels. Thus, at least in neuroblastoma cells, TH expression is controlled in an 'all or nothing' manner. The usage of isoform-specific antibodies may also thus provide further clues in studying the TH distribution in human tumor tissue.
Finally, as discussed by the authors of this paper (1), it is now extremely interesting to further elaborate on mechanisms by which enzyme gene expression occurs. Since the data clearly point to a genomic control of TH activity rather than to feed-back control, it now remains to be determined what (tumor-specific) factors affect TH mRNA levels on the genomic levels. Several studies, mainly using $\mathrm{PC} 12$ cells, have been performed to investigate $\mathrm{TH}$ gene regulation. It has been shown that cAMP, glucocorticoids, phorbol esters or calcium ionophores stimulate endogenous TH gene transcription and/or TH gene promoter activity. In a recent and very elegant study, the muscarinic M1-acetylcholine receptor agonist carbachol has been shown to interact with the TH gene promoter (7). This is mainly mediated by a cAMP response element (CRE), since mutagenization at the CRE abolishes the response to carbachol by approximately $60 \%$. Also, the response of the $\mathrm{TH}$ gene promoter to carbachol appears to be at least partially mediated by transcription factors interacting with the TH CRE. The activating protein 1 (AP1) site appears to be less important in this response. It is also clearly conceivable that other mediators including tumor-specific growth factors such as IGFs-I and -II and proto-oncogenes such as c-fos or c-myc play an important role in this context. Increases in second messengers along the signal transduction pathway will involve the activation of protein kinase $\mathrm{C}$ and a calmodulin kinase. The stimulation of these pathways will lead to the activation of transcription factors that bind to and activate CRE and AP1 sites within the TH gene 5'-flanking region. Further studies are clearly needed to address these exciting questions and to elucidate the mechanisms upstream of an enhanced TH gene expression.

In summary, the data from this and other studies clearly corroborate not only an enhanced activity of TH and other catecholamine synthesizing enzymes in pheochromocytoma tissue, but also very convincingly demonstrate that enhanced catecholamine content and enzyme activity is secondary to alterations at the genomic levels, i.e. enhanced TH mRNA expression. On a more pathophysiological level, these findings have clearly provided a stimulating basis for further investigating the role of TH mRNA expression in controlling catecholamine secretion, and also for further analysing the mechanisms and factors governing this expression at the DNA level. The clinical perspectives on the other hand can also be put straightforwardly: tumor-specific expression of enzyme mRNA will further allow an explanation not only for the varying clinical phenotypes, but also for the differentiation of this tumor into a presumably benign or malignant type. In addition to the latter characterization, the differences in mRNA levels according to the sporadic versus familial character of the tumors may also provide important clues to the pathogenesis of diseases such as MEN of vHLS. Finally, differences in growth factors and oncogenes 
involved, as well as in the activation of signal transduction pathways, may account for variable enzyme mRNA expression. Thus, there will be new and exciting perspectives in pheochromocytoma research, spanning the whole range from novel molecular to clinical findings.

\section{References}

1 Isobe K, Nakai T, Yukimasa N, Nanmoku T, Takekoshi K \& Nomura F. Expression of mRNA coding for four catecholaminesynthesizing enzymes in human adrenal pheochromocytomas. European Journal of Endocrinology 138 383-387.

2 Kopf D, Bockisch A, Steinert H, Hahn K, Beyer J, Neumann HPH et al. Octreotide scintigraphy and catecholamine response to an octreotide challenge in malignant pheochromocytoma. Clinical Endocrinology 199746 39-44.

3 Mulligan LM, Eng C, Healey CS, Clayton D, Kwok JB, Gardner E et al. Specific mutations of the RET proto-oncogene are related to disease phenotype in MEN 2A and FMTC. Nature Genetics 1994 $670-74$.

4 Hollenbach E, Schulz C \& Lehnert H. Rapid and sensitive determination of catecholamines and the metabolite 3-methoxy4-hydroxy-phenylglycol (MHPG) using HPLC following a novel extraction procedure. Life Sciences 1998 (In Press).

5 Lehnert H, Reinstein DK, Strowbridge BW \& Wurtman RJ. Neurochemical and behavioral consequences of an acute uncontrollable stress: effects of dietary tyrosine. Brain Research $1984303215-223$.
6 El-Mestikaway S, Glowinski J \& Hamon M. Tyrosine hydroxylase activation in depolarized dopaminergic terminals - involvement of $\mathrm{Ca}^{2}$-dependent depolarization. Nature $1983302830-$ 832 .

7 Chen Y, Best JA, Nagamoto K \& Tank AW. Regulation of tyrosine hydroxylase gene expression by the $\mathrm{m} 1$ muscarinic acetylcholine receptor in rat pheochromocytoma cells. Molecular Brain Research $19964042-54$.

8 Wurtman RJ \& Axelrod J. Control of enzymatic synthesis of adrenaline in the adrenal medulla by adrenal cortical steroids. Journal of Biological Chemistry 1966241 2301-2305.

9 Kimura N, Miura Y, Nagatsu I \& Nagura H. Catecholamine synthesizing enzymes in 70 cases of functioning and nonfunctioning pheochromocytoma and extra-adrenal paraganglioma. Virchows Archiv. A, Pathological Anatomy and Histopathology 1992421 25-32.

10 Tümer D, Brown JW, Carbaleira A \& Fishman LM. Tyrosine hydroxylase gene expression in varying forms of human pheochromocytoma. Life Sciences 199659 1659-1665.

11 Funahashi H, Imai T, Tanaka Y, Tobinaga J, Wada M, Matsuyama $\mathrm{T}$ et al. Discrepancy between PNMT presence and relative lack of adrenaline production in extra-adrenal pheochromocytoma. Journal of Surgical Oncology 199457 196-200.

12 Haycock JW. Multiple forms of tyrosine hydroxylase in human neuroblastoma cells: quantitation with isoform-specific antibodies. Journal of Neurochemistry 199360 493-502.

Received 10 December 1997

Accepted 15 December 1997 